\title{
Superconducting microwave cavities as quantum nanomechanical transducers
}

\author{
G. J. Milburn ${ }^{1}$, M. J. Woolley ${ }^{1}$, A. C. Doherty ${ }^{1}$ and K. C. \\ Schwab $^{2}$ \\ ${ }^{1}$ School of Physical Science, The University of Queensland, QLD 4072 \\ ${ }^{2}$ Cornell University, Ithaca, NY 14853 USA \\ milburn@physics.uq.edu.au
}

\begin{abstract}
We show how a superconducting coplanar microwave cavity can be used as a quantum limited displacement transducer for a nanomechanical resonator by demonstrating that nanomechanical squeezing can be detected in the cavity field.
\end{abstract}

We propose a scheme for quantum limited detection of the displacement of a nanomechanical cantilever [1] using a microwave cavity, in the form of a superconducting coplanar waveguide [2], and homodyne detection scheme. As a test of the scheme we show that we can detect mechanical squeezing of the nanomechanical resonator when it is driven parametrically.

Suppose the cavity has resonant frequency $\omega_{c}$, and the cantilever has resonant frequency $\nu$ and mass $m$. The uncoupled cavity may be described by an equivalent inductance $L$ and equivalent capacitance $C$. The capacitive coupling between the cavity and input transmission line, as a function of cantilever position $x(t)$, is added in parallel to the equivalent capacitor and may be approximated by $C_{0}(x)=C_{0}(1-x(t) / d)$ where $C_{0}$ represents an equilibrium capacitance and $d$ is the equilibrium cantilever to cavity separation. Thus the coupled cavity has an equivalent capacitance $C_{\Sigma}=C+C_{0}$, such that $\omega_{c}=1 / \sqrt{L C_{\Sigma}}$. The capacitive energy of the system can then be given by $Q^{2} /\left(2 C+2 C_{0}(x)\right)=Q^{2} /\left(2 C_{\Sigma}\right)+\left(\beta / 2 d C_{\Sigma}\right) x(t) Q^{2}$ where $\beta=C_{0} / C_{\Sigma}$.

The cavity is driven by the electric potential $e(t)=\sqrt{2 \hbar \omega_{c} L} \epsilon\left(e^{i \omega_{d} t}+e^{-i \omega_{d} t}\right)$. Parametric driving of the cantilever at frequency $\Omega$ with strength $\chi$ is a modulation of the cantilever's spring constant and hence may be given by $k(t)=4 m \nu\left(\chi^{*} e^{i \Omega t}+\chi e^{-i \Omega t}\right)$. Hamiltonian for the coupled system is

$$
H=\frac{p^{2}}{2 m}+\frac{m \nu^{2} x^{2}}{2}+\frac{\Phi^{2}}{2 L}+\frac{Q^{2}}{2\left(C+C_{0}(x)\right)}-e(t) Q+\frac{k(t)}{2} x^{2}
$$

We can now quantise the Hamiltonian, promoting the canonical coordinates to operators with $[\hat{x}, \hat{p}]=$ $[\hat{Q}, \hat{\Phi}]=i \hbar$. The quantum Hamiltonian may be expressed in terms of the raising and lowering operators for the cavity $\left(a^{\dagger}, a\right)$ and the resonator canonical operators $(\hat{q}, \hat{p})$. Typically, $\omega_{c} \gg \nu$ since $\omega_{c} / 2 \pi \approx 6 \mathrm{GHz}[3]$, while $\nu / 2 \pi \approx 20 \mathrm{MHz}$ [4]. It is convenient to move into an interaction picture with respect to $\hbar \omega_{d} a^{\dagger} a+\hbar \nu b^{\dagger} b$, and neglect terms oscillating at $\pm 2 \omega_{d}$. The resulting Hamiltonian is

$$
H_{I}=\hbar \delta a^{\dagger} a+\hbar \kappa \hat{X}(t) a^{\dagger} a+\hbar \epsilon\left(a+a^{\dagger}\right)+\hbar\left(\chi^{*} b^{2}+\chi b^{\dagger 2}\right)
$$

where $\delta=\omega_{c}-\omega_{d}$. The coupling term represents a low frequency modulation of the cavity resonance frequency. This will cause a phase modulation of the cavity field and write sidebands onto the cavity spectrum at multiples of $\nu$ from $\omega_{c}$.

As the cavity $\mathrm{Q}$ is very large, we can decompose the field into sideband modes, $a(t)=a_{0}(t)+a_{+}(t) e^{-i \nu t}+$ $a_{-}(t) e^{i \nu t}$, where - refers to the blue sideband and + refers to the red sideband. We now can choose the driving frequency for the cavity to be resonant with the red, with $\delta=-\nu$, or blue, with $\delta=\nu$, sideband. Then linearising the dynamics around the corresponding steady states, we find that the field dynamics at the respective sidebands are described by the effective Hamiltonians,

$$
\begin{aligned}
& H_{r}=\hbar g\left(a_{+}^{\dagger} b+a_{+} b^{\dagger}\right) \\
& H_{b}=\hbar g\left(a_{-}^{\dagger} b^{\dagger}+a_{-} b\right)
\end{aligned}
$$

where $g=\kappa \epsilon / \nu$, The cavity is damped at $\mu$ and the nanomechanical resonator has damping rate $\gamma$. Using standard methods [5] we can now calculate the normally ordered squeezing spectrum, $S(\omega)$, in the sideband 


\section{JMA2.pdf}

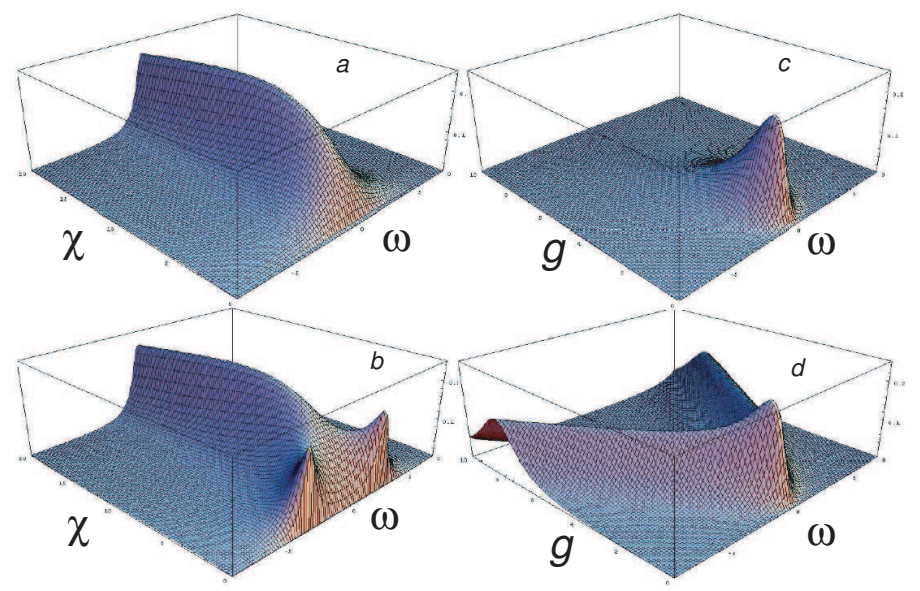

Fig. 1. Negated squeezing spectra as a function of $\chi$ for $g=3$ for (a) driving on the blue sideband and (b) driving on the red sideband. Negated squeezing spectra as a function of $g$ for $\chi=1$ for (c) driving on the blue sideband and (d) driving on the red sideband. All parameters scaled to $\mu$, the cavity decay.

modes. In figure 1 we plot $1-S(\omega)$, versus parametric driving (a,b) and coupling (c,d) for blue and red sidebands. If this quantity is positive then noise is reduced below the vacuum level. We have taken the following physically reasonable parameters, $\omega_{c} / 2 \pi=6 \mathrm{GHz}, \nu / 2 \pi=20 \mathrm{MHz}, \gamma=0.126 \times 10^{4} \mathrm{~s}^{-1}$ and $\mu=$ $0.38 \times 10^{6} \mathrm{~s}^{-1}$. Assuming a mass of $m=10^{-15} \mathrm{~kg}$ and $d=100 \mathrm{~nm}$ we get $\beta=0.002$. The effective coupling constant $g$ depends on the steady state cavity field. This can be made large, but should not be made so large that voltage breakdown on the cavity occurs.

\section{References}

1. A.N. Cleland and M.L. Roukes, Appl. Phys. Lett. 69, 2653 (1996).

2. L. Frunzio, A. Wallraf, D. Schuster, J. Majer and R. Schoelkopf, IEEE Trans. on Appl. Super. 15, 860 (2005).

3. A. Wallraff et al., Nature 431, 162 (2004).

4. M.D. LaHaye et al., Science 304, 74 (2004).

5. D.F. Walls and G.J. Milburn, Quantum Optics, 2nd edt., (Springer, Berlin, 2007). 